\title{
Characterization of Otto Chips by Particle Swarm Optimization
}

\author{
Adonias Luna Pereira da Silva ${ }^{1}$, Sérgio Campello Oliveira ${ }^{1}\left(\mathbb{D}\right.$, Gustavo Oliveira Cavalcanti ${ }^{1}$ (D), \\ Manoel Alves de Almeida Neto ${ }^{1}$ (D), Maria Renata Nascimento dos Santos ${ }^{1}$ (D), Ignacio \\ Llamas-Garro $^{2}$ (D) Jung-Mu Kim ${ }^{3}$ (D), Gabriel de Freitas Fernandes ${ }^{4}$ (D), Eduardo Fontana ${ }^{4}$ (D) \\ ${ }^{1}$ Escola Politécnica, Universidade de Pernambuco, Recife, Brasil, alps@ecomp.poli.br, sergio.campello@upe.br, \\ gustavooc@poli.br,maan@ecomp.poli.br,marenasa.18@gmail.com \\ ${ }^{2}$ Centre Tecnològic de Telecomunicacions de Catalunya, Castelldefels, Spain, ignacio.llamas@cttc.es \\ ${ }^{3}$ Division of Electronic Engineering, Jeonbuk National University, Jeonju, Republic of Korea, \\ jungmukim@jbnu.ac.kr \\ ${ }^{4}$ Dep. de Eletrônica e Sistemas, Universidade Federal de Pernambuco, Recife, Brasil, gabriel.freitasf@ufpe.br, \\ fontana@ufpe.br
}

\begin{abstract}
Recently a surface plasmon resonance (SPR) optical sensor, based on the Otto configuration - the Otto chip - has been developed. One essential step in the quality control of the fabrication process is characterization of the active region of several devices in a batch. Characterization is done by measuring the angular spectrum of the optical reflectance on several points across the active region of the device, and determining parameters by regression analysis of the data. Traditional gradient methods used in the regression process are extremely dependent on an initial guess and are not very efficient for batch analysis of curves, when those include poorly defined SPR spectra, where an initial guess may be hard to infer. An alternative approach for the regression problem is to model the analysis as an optimization problem and using an efficient stochastic algorithm. In this paper one discusses the use of Particle Swarm Optimization (PSO) for characterization of Otto chip devices. From comparative studies carried out in an existing Otto chip, it is observed that PSO can be a very efficient approach for batch analysis and yields better results when compared with the traditional gradient-based regression method.
\end{abstract}

Index Terms - particle swarm optimization, surface plasmon resonance, SPR, Otto chip, PSO, regression analysis

\section{INTRODUCTION}

Surface Plasmon Resonance (SPR) [1] can be observed in the optical reflectance function of a metal surface, in a specially configured multilayer configuration. The effect is highly sensitive to changes of the optical characteristics of the medium adjacent to the metal, and because of this, SPR has proven to be an excellent tool for optical sensing [2], [3], and it has been applied in the construction of biological sensors [4]-[7], and gas sensors [8], [9], for example.

The multilayer structure for SPR excitation can be built with different configurations, including Otto's [1] and Kretschmann's [10]. Recently, a device based on the Otto configuration — the Otto chip [11] was developed, with inherent advantages over Kretschmann's. This is because, differently than the latter, the Otto chip can be fabricated on CMOS compatible silicon substrates, being amenable to integration with microcircuits, thus enabling development of new sensor architectures. Another advantage is that 
Otto chip devices have true micron size flow channel heights, therefore decreasing sample volumes, thus allowing integration to silicon microfluidic circuits as well [11].

Given the growing interest on the Otto chip for optical sensing, one important step in using these devices is establishing reliable characterization tools for quality control of the response of fabricated devices. A key element in the quality assessment is precise characterization of the gap uniformity of the active region of the chip. The first step in the characterization is done by measuring the SPR effect within the angular spectra of the reflectance functions on different points across the active region. From the experimental data, regression procedures are required to find the gap profile and surface optical parameters.

Currently, the procedure applied for characterization is the traditional, gradient-based, regression analysis [12]. However, this method is totally dependent on an initial estimate of the parameter values [13], and this estimate must be close enough to the expected values, because otherwise convergence of the algorithm is not achieved. Thus, in points on the surface for which the SPR effect is poorly defined or even absent, it is not possible to have good estimates for the initial values, and no convergence is obtained. As a result, one is not able to establish a procedure for automatic characterization of a large number of data sets across the device's active area, making it difficult carrying out single device characterization, and even worse, of several devices in a batch.

An alternative approach for the regression problem is to model the analysis as an optimization problem and using an efficient optimization algorithm. This alternative has already been applied on the extraction of optical constants for Kretschmann's configuration [14]-[17]. In [14] the constants were obtained by minimizing the root mean square error of the experimental and calculated reflectance, using particle Particle Swarm Optimization (PSO) [18], a well-known method for optimization problems. The focus of the present paper is to apply a similar approach for the nonlinear regression analysis of Otto chip devices [19], and to determine to what extent this strategy can be efficient and amenable to large scale device characterization once, as further explained, nonlinear regression analysis on Otto's configuration have to deal with two sets of parameters as possible solutions.

PSO is a computing algorithm inspired by the flocking of birds [18]. It has been already used before for solving regression problems with good results [20]. Unlike the gradient-based approach, PSO does not require determining an initial estimate of parameters and consequently can be used to automate the regression for a large number of data sets, as demonstrated in the following sections.

\section{REFLECTION FunCTION IN THE OtTO CONFIGURATION}

\section{A. Surface Plasmon Resonance}

The SPR effect can be observed in metallized optical fibers [21], [22], on metal diffraction gratings [23] and under frustrated total internal reflection within a prism [1], [10]. Observation is done by illuminating the prism with a plane-polarized, monocrhomatic, light beam, and measuring the horizontal face reflectance as a function of the incidence angle $\theta$, as illustrated in Fig.1.

In Kretschmann's prism coupling configuration, which is similar to the scheme of Fig.1, the illuminated prism horizontal face, shown in the figure, would be directly coated with a thin metal film [10]. In the Otto scheme of Fig.1, the illuminated prism face is separated from a thick metal film by a narrow gap [1]. 


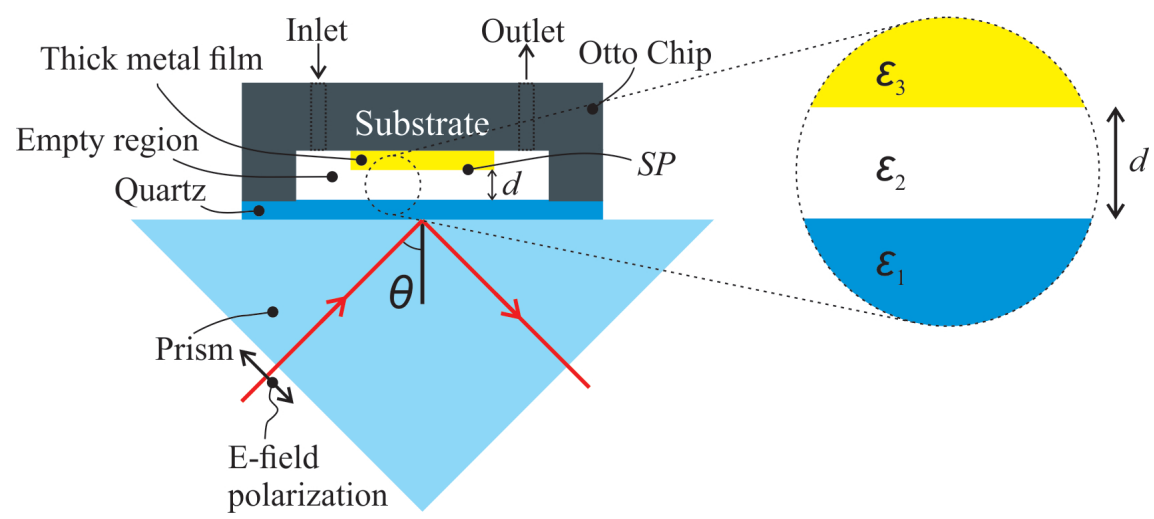

Fig. 1. Otto's prism configuration scheme for coupling an incoming light beam to a surface plasmon on the interface of a thick metal film.

For plane polarized light, the internal reflectance of the prism's horizontal face, in the scheme of Fig.1, with two interfaces separating media 1 (glass region), 2 (gap region) and 3 (metal region), with permittivities $\epsilon_{1}, \epsilon_{2}$ and $\epsilon_{3}$, respectively, is given by [13]

$$
R_{\text {theo }}=\left|\frac{r_{1,2}+r_{2,3} \exp \left(-2 j k_{2} d\right)}{1+r_{1,2} r_{2,3} \exp \left(-j 2 k_{2} d\right)}\right|^{2} .
$$

The $r$ coefficients are given by [13]

$$
r_{i, i+1}=\frac{\varepsilon_{i+1} k_{i}-\varepsilon_{i} k_{i+1}}{\varepsilon_{i+1} k_{i}+\varepsilon_{i} k_{i+1}},
$$

with $i=1,2$, and the wavenumbers $k_{i}$ are

$$
k_{i}=k_{0}\left[\varepsilon_{i}-\varepsilon_{1}(\sin \theta)^{2}\right]^{\frac{1}{2}},
$$

with $i=1,2$ and 3 and $k_{0}=2 \pi / \lambda$ at the wavelength $\lambda$.

\section{B. The Otto chip}

Figure 2 illustrates qualitatively the shape of two distinct reflectance curves given by (1) as a function of the incidence angle $\theta$. In this figure, $\theta_{T I R}$ is the angle of total internal reflection and $\theta_{S P}$ is the SP excitation angle, for a fixed set of multilayer parameters. Variation in the permittivitty $\epsilon_{2}$ of the gap

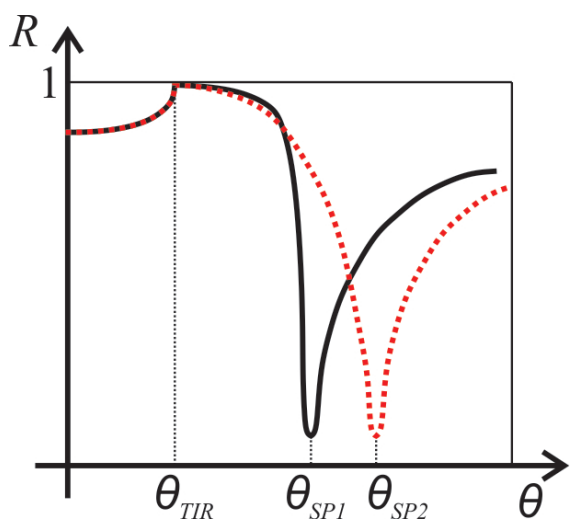

Fig. 2. Qualitative plots of the reflectance function given by (1) for two sets of multilayer parameters in the Otto configuration of Fig.1. 
region, e.g. by subjecting it to a variable gas concentration or to a flowing liquid with varying refractive index, produces a shift in the SP excitation angle. This is illustrated qualitatively in Fig.2 by a shift from $\theta_{S P 1}$ to $\theta_{S P 2}$. Because of the sharpness of the resonance curves, which occurs for noble metals, this angular shift yields large reflectance changes, and this is the main feature of the SPR effect that has attracted so much attention for the development of optical sensors.

In this paper one uses the model function in (1) to determine the gap thickness $d$ and the metal permittivity $\epsilon_{3}$, illustrated in Fig.1 across the active area of an Otto chip device [11]. A simplified diagram of the device is illustrated in Fig.3. Each chip is fabricated on a silicon substrate, having an etched cavity $2.5 \mu \mathrm{m}$ deep, over which there is an $1 \mathrm{~cm} \times 1 \mathrm{~cm}$ gold film, $300 \mathrm{~nm}$ thick. This cavity is sealed with a quartz window and in the final configuration, the gap between gold and quartz surfaces is approximately $2.2 \mu \mathrm{m}$. On the silicon side of the chip, there are two etched holes through which one can flow either gaseous or liquid substances. The quartz window of the Otto chip can then be placed in optical contact with the prism in the scheme illustrated in Fig.1, for characterization.

The Otto chip, illustrated in Fig.3, was previously characterized using an automated reflectometer [24], that operates on the measurement principle illustrated in Fig.1. The light source in the reflectometer is a diode laser tunned in $980 \mathrm{~nm}$, and the prism is made of BK7 glass. The reflectometer has attributes of two-dimensional scanning of different points on the chip's active region. For the measurement of the angular dependence of the reflectance, the incidence angle is swept in a range of $6 \mathrm{deg}$, with a resolution of approximately $0.017 \mathrm{deg}$, yielding 350 reflectance values per illuminated point on the surface. A total of 121 surface coordinates were measured on a square grid of 11 x 11 corresponding to an area larger than the $1 \mathrm{~cm} \times 1 \mathrm{~cm}$ metallized region on the Otto Chip cavity.

For each point measured on the chip, the reflectometer has computer controlled automatic adjustments to maintain the laser footprint stationary on the surface, while the incidence angle $\theta$ is changed [25]. The complete set of measured data is available in the literature [26] and can be directly visualized in the video of [27]. The video allows identifying the metallized region, areas corresponding to silicon/quartz bonds and edges between silicon and gold on the cavity region. The gold coated region of the chip exhibits well defined resonances.

One typical measured SPR effect is shown in Fig.4. As can be observed in the figure, the measured SPR curve has a lorentzian type absorption profile with a full-width-at-half-maximum (FWHM) of approximately $0.2 \mathrm{deg}$. As shown in more detail in the upcoming section and in the video of ref. [27], distinct shapes of the SPR effect are observed.

Characterization of the Otto chip, after it has been fabricated, is an important quality control task, and requires retrieving the gap thickness and gold optical constants across the active area of the chip, by regression of the experimental data. Traditionally a gradient based nonlinear regression algorithm is employed to determine parameters. The approach requires setting initial values for the parameters to be obtained. It has been previously shown that the lorentzian-type line shape of the reflectance curve leads to two sets of initial values for the metal complex permittivity and gap thickness [13], [28]. The regression analysis algorithm, after completed, thus yields two sets of parameters as final solutions of the problem. This ambiguity is hard to resolve, and has to be raised by either comparing the obtained values of the complex permittivity with known values tabulated in the literature or, alternatively, by analyzing a wider angular range of the reflectance data, in conjunction with a heuristic approach for parameter determination, such as that employed in this paper. 


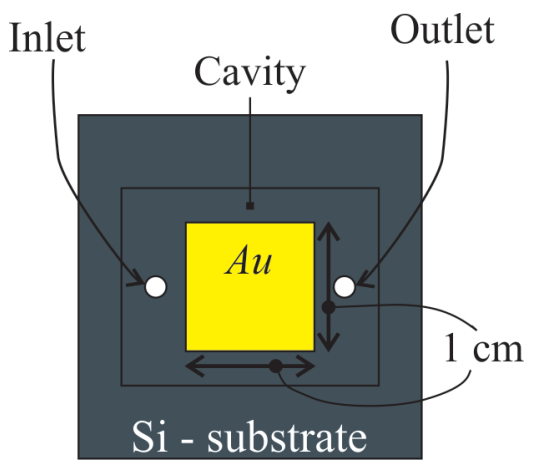

Fig. 3. Simplified illustration of an Otto chip device [11].

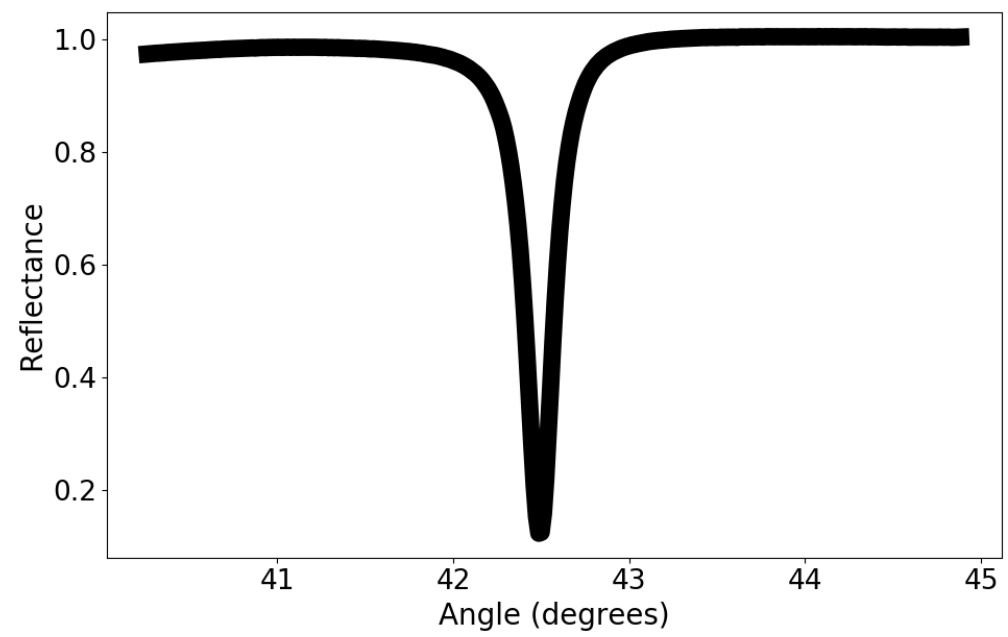

Fig. 4. Typical SPR measured data for the Otto chip at $\lambda=975.1 \mathrm{~nm}$. Notice that at this wavelength, and for gold, the SPR effect has a very narrow FWHM (full-width-at-half-maximum), of approximately $0.2 \mathrm{deg}$.

\section{Particle Swarm Optimization (PSO)}

Particle swarm optimization (PSO) is a swarm intelligence algorithm inspired by the flocking behavior of birds, proposed in 1995 by James Kennedy and Russell Eberhart [18]. The particles are distributed on a search space and their positions represent possible solutions to the function to be optimized. In this work, each particle is the 3 -element, parameter vector,

$$
\vec{X} \equiv\left(\varepsilon^{\prime}, \varepsilon^{\prime \prime}, d\right)
$$

with $\varepsilon^{\prime}$ and $\varepsilon^{\prime \prime}$ representing, respectively, the real and imaginary parts of the gold complex permittivity, i.e.,

$$
\varepsilon_{3} \equiv \varepsilon^{\prime}+j \varepsilon^{\prime \prime},
$$

and $d$, the gap thickness.

The domain search space is constituted by variables of the function under optimization. In order to find better solutions, the particles' positions are updated iteratively according to [18]

$$
\vec{X}_{i+1}=\vec{X}_{i}+\vec{V}_{i+1}
$$

where $\vec{V}_{i}$ and $\vec{X}_{i}$ are the velocity and position vectors, respectively, calculated for each one of the $N$ 
particles of the swarm at the $i$-th iteration. Time is considered unitary.

The swarm communication strategy determines how the particles will influence each other's velocity. On the global topology communication strategy, applied in this work, the particles have their movements guided by the best position (solution) found by the whole swarm $\vec{G}_{\text {best }}$ and their self best position $\vec{P}_{\text {best }}$. The velocity is then calculated for each particle by [29]

$$
\vec{V}_{i+1}=\omega \vec{V}_{i}+c_{1} r_{1}\left(\vec{P}_{\text {best }}-\vec{X}_{i}\right)+c_{2} r_{2}\left(\vec{G}_{\text {best }}-\vec{X}_{i}\right)
$$

where $\omega$ is the inertia weight $r_{1}$ and $r_{2}$ are random weights generated in every iteration for each particle in the domain $[0,1]$ and $c_{1}, c_{2}$ are the cognitive constants, both of them set to 2.05 according to [30]. The inertia weight influences on the PSO algorithm convergence.

Another strategy investigated in this work was that proposed by Clerc and Kennedy [30]. In this strategy a constriction factor is applied to all terms of the velocity equation

$$
\vec{V}_{i+1}=\chi\left(\vec{V}_{i}+\varphi_{1}\left(\vec{P}_{\text {best }}-\vec{X}_{i}\right)+\varphi_{2}\left(\vec{G}_{\text {best }}-\vec{X}_{i}\right)\right),
$$

where

$$
\varphi_{1}=c_{1} r_{1}
$$

and

$$
\varphi_{2}=c_{2} r_{2}
$$

In (8), $\chi$ is the constriction factor and was set to 0.7296 [30]. As further described, Clerc approach returns better results. The algorithm execution procedure under this strategy is summarized in the pseudocode listed in Algorithm 1.

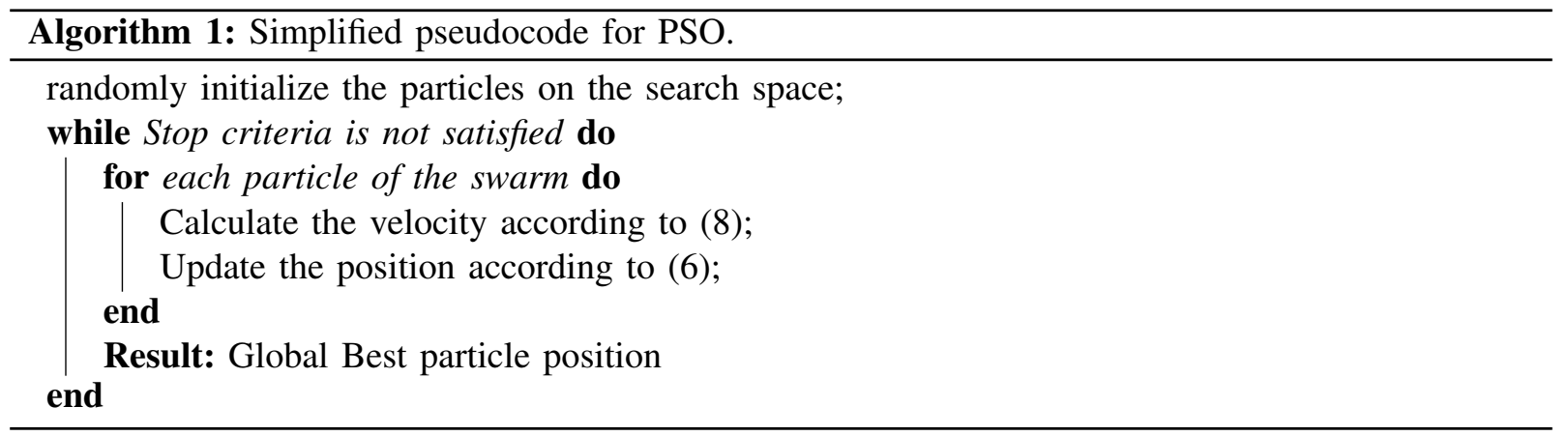

\section{REGRESSION ANALYSIS BY PSO}

As described in Section II-B, 121 reflectance curves were obtained by scanning the laser footprint across the cavity area of the Otto chip. From those, a case study was conducted with 20 curves, in which the SPR effect was clearly observed, as described next.

\section{A. Error function}

The regression using PSO is done by optimizing the material parameters of the Otto chip, through minimization of the average absolute error between measured data and the theoretical model function given by (1). As indicated in (1), for a fixed wavelength, the reflectance is dependent on the three 
permittivity parameters $\varepsilon_{1}, \varepsilon_{2}, \varepsilon_{3}$, on the gap thickness $d$ and on the incidence angle $\theta$. The prism glass permittivity $\varepsilon_{1}$, which is very close to the quartz window permittivity of the Otto chip, is well defined, and the gap region permittivity is that of air, i.e., $\varepsilon_{2}=1$. Thus, as pointed out earlier, the parameters to be optimized are the elements of vector $\vec{X}$ defined in (4).

In the measurement wavelength, the condition $\varepsilon^{\prime}<0$ is satisfied and for gold, the condition $\left|\varepsilon^{\prime \prime}\right|<<$ $\left|\varepsilon^{\prime}\right|$ is also satisfied. With the parameter definitions thus defined, and given that the number of reflectance values per measured surface point is constant, the error function can be written in the form

$$
\Delta(\vec{X})=\sum_{j=1}^{J}\left(R_{\text {theo }}\left(\theta_{j}, \vec{X}\right)-R_{j}\right)^{2},
$$

where $J=350$ is the number of incidence angles measured in the experiment and $R_{j}$ is the experimental reflectance at the incidence angle $\theta_{j}$.

For the gradient-based nonlinear regression method, fully described in matrix form in ref. [12], a gradient search method is implemented to determine the parameter vector (4) that minimizes the error function (11). The minimization condition is obtained by setting

$$
\vec{\nabla}_{X} \Delta=0,
$$

with

$$
\vec{\nabla}_{X} \equiv\left(\frac{\partial}{\partial \varepsilon^{\prime}}, \frac{\partial}{\partial \varepsilon^{\prime \prime}}, \frac{\partial}{\partial d}\right)
$$

representing the nabla operator in the parameter space. As the model function is nonlinear in the elements of $\vec{X},(12)$ has to be solved iteratively, and at each iteration step, the model function (1) is linearized relative to the elements of the parameter vector $\vec{X}$, as detailed in ref. [12]. By doing this, (12) can be cast as a set of three linear equations in the corrections to each element of the parameter vector. The procedure is run until the maximum magnitude of the correction vector at the $k$-th iteration,

$$
\delta \vec{X}_{k} \equiv\left|\vec{X}_{k+1}-\vec{X}_{k}\right|
$$

is smaller than a given error parameter.

As pointed out in Section II-B, for the case of SPR curves, it has been shown previously that by use of a lorentzian approximation to the waveshape, two initial guesses can be determined analytically for vector $\vec{X}$. The two sets are represented in this paper by the binary variable $s= \pm 1$. The gradient-based iterative algorithm uses those initial estimates for $\vec{X}$ to determine two solutions to the problem.

\section{B. Determination of the PSO parameters}

The efficiency of the PSO procedure on finding a global optimum is influenced by the algorithm configuration. Through experiments it is possible to determine adequate parameters for the algorithm, and in most cases one can find the overall best solution. In this paper, the influence on the algorithm efficiency was investigated for the following parameters: number of particles, number of iterations and inertia strategy.

In (7) and (8) it is clear that the inertia weight and the constriction factor will determine the influence of the previous velocity on the calculation of the current position. This factor regulates the two known search stages of the swarm: exploration, the global search, and exploitation, the local search. Therefore 
it is important to find a balance between these two stages of the swarm search so that the algorithm can converge to the overall optimum. In this work one examined the following inertia strategies: constant [29] for $w=0.5,0.7$ and 0.9, random [30], simulated annealing [31], chaotic [32] and linear decreasing [33]. As already described, the Clerc strategy was also investigated [30]. It was observed that the algorithm configured with 500 particles and Clerc strategy resulted in the least variance and average error when compared to other inertia strategies.

In order to confirm that the Clerc strategy had the best performance of PSO configurations, we applied the non-parametric Wilcoxon hypothesis test [34], because the Kolmogorov - Smirnov test indicated that the results did not fit in a Normal Distribution [35]. All configurations were compared with each other considering a confidence level of $95 \%$. The results obtained by the tests are presented in Table I. For the case of constant inertia, only the cases $w=0.5$ and $w=0.7$ were included in the test, as shown in Table I, as they yielded the best results. In the table, $h_{0}$ represents the probability obtained assuming both configurations being equal, whereas, $h_{1}$, the probability that the first configuration yields an average error larger than that of the second one. It is clear from Table I that the Clerc strategy has the best overall performance.

TABLE I. Wilcoxon STATISTIC ANALYSIS.

\begin{tabular}{cccc}
\hline Configuration 1 & Configuration 2 & $h_{0}$ & $h_{1}$ \\
\hline$w=0.7$ & $w=0.5$ & 0.005223 & 0.997389 \\
$w=0.7$ & Clerc & 0.010396 & 0.994802 \\
$w=0.7$ & Linear & 0.305390 & 0.847305 \\
$w=0.5$ & Clerc & 0.120649 & 0.939676 \\
$w=0.5$ & Linear & 0.046850 & 0.023425 \\
Clerc & Linear & 0.026703 & 0.013352 \\
\hline
\end{tabular}

To determine the number of iterations needed to achieve proper convergence, an analysis was made on the percentage reduction of the error of the best particle of the swarm over the iterations, as shown in Fig.5. As can be noticed from the plot, the average error reductions over 100 iterations indicates that the algorithm does not reduce significantly the error after the 30th iteration.

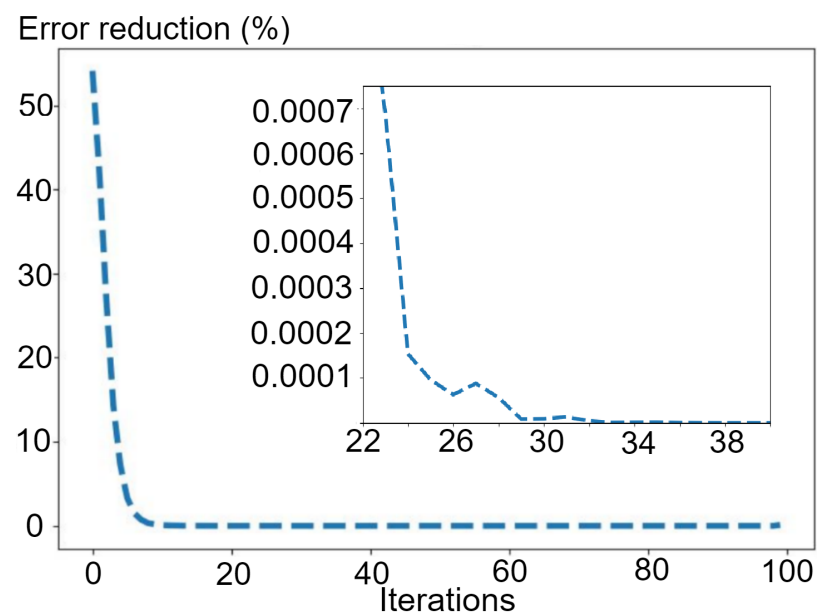

Fig. 5. Error reduction rate versus number of iterations using PSO with 500 particles and Clerc. The inset plot shows a magnified vertical scale in the iteration range between 22 and 38. 


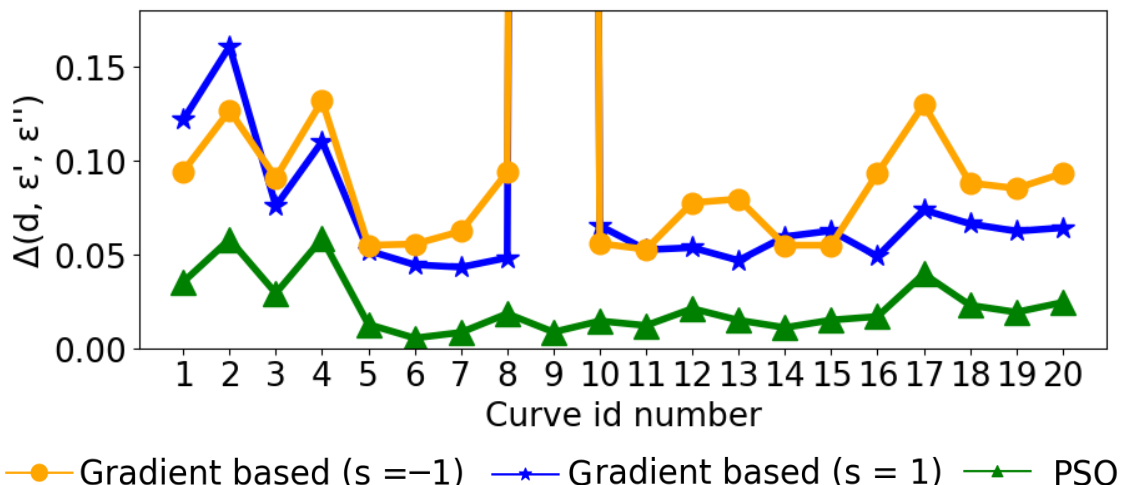

Fig. 6. Average absolute error function, as defined in (11), between measured data and the theoretical model function (1) for the distinct Otto chip points. Point number nine presents errors of $4.8382(s=-1)$ and $4.8951(s=1)$ for gradient solutions and 0.0086 for PSO.

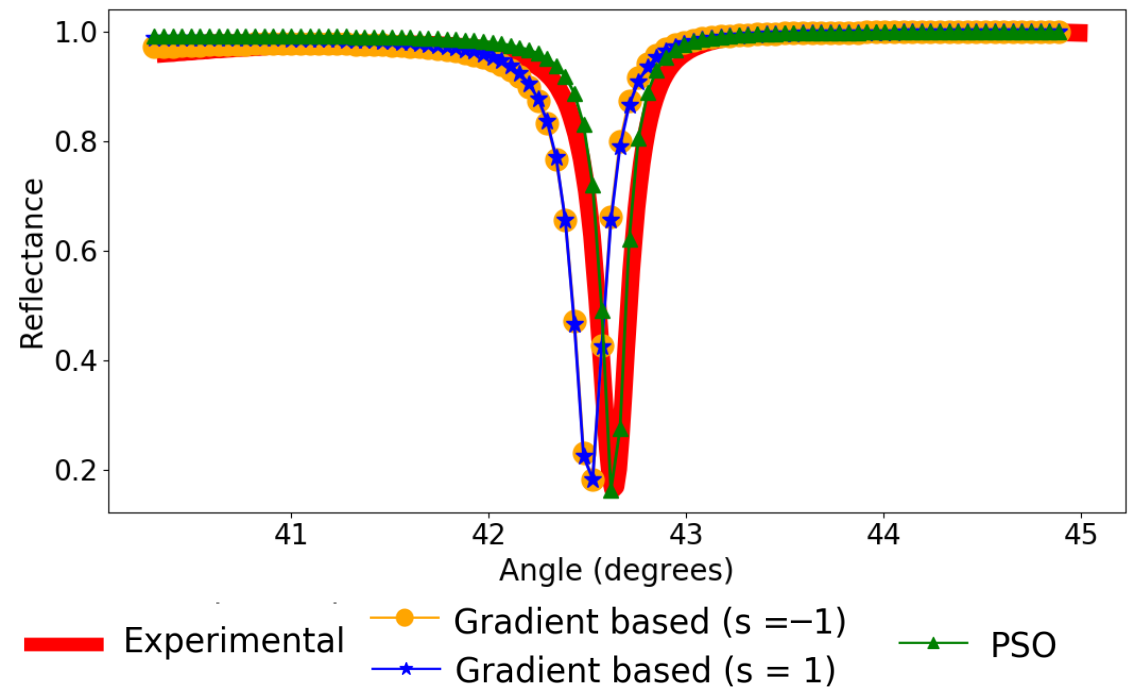

Fig. 7. Experimental SPR reflectance and theoretical regression curves for point number nine of the Otto chip.

\section{RESULTS}

The use of PSO on the regression of SPR curves, through minimization of the error function (11), yielded smaller average absolute errors. Once PSO has random parameters in its definition, for each point on the Otto chip, the PSO algorithm was executed 100 times. Each execution was done with 500 particles, 30 iteration and using Clerc's velocity equation (8). PSO, in its best execution, was able to find an error 10.27 times smaller than that of the gradient method. For point number 13, for example, all 100 executions of PSO found smaller average errors than those found by the gradient-based technique.

Figure 6 shows the three average absolute errors after optimization, two of them found by the gradient based approach and the other by PSO, for all 20 Otto chip point analyzed in this paper. The sign of the $s$ parameter $( \pm 1)$ labels a given solution of the gradient method. As can be noticed from the plots, the PSO approach exhibits smaller absolute errors than the gradient-based solutions, overall. Point 9 on the Otto chip yielded off-the-chart errors for the two gradient solutions. For this point, the absolute errors were: $4.8382(s=-1)$ and $4.8951(s=1)$. On the other hand, the error for PSO was only 0.0086.

Figure 7 exhibits the SPR experimental data and all three predictions from the regression analysis, taking point 9 as an example. It is noticed that the gradient solutions converged to a local minimum 
and missed the global minimum error. On the other hand, the plot shows that PSO was able to find the global minimum error. This shows one of the problems of the gradient based approach, i.e., the regression procedure may be trapped, in certain situations, to local minima, and automation of the procedure may become difficult. Occurrences of local minima in the gradient method are common when random errors are present, e.g., due to the existence of local defects or micro-particles on the measured surface point, which may affect the location and shape of the measured SPR curve. This in turn may produce deviations of the initial guess from the final solution, facilitating convergence to a local minimum. PSO on the other hand appears to be more feasible in finding the global solution and thus can be configured for automated analysis.

Figure 8 exhibits the optimum values obtained for $\varepsilon^{\prime}, \varepsilon^{\prime \prime}$ and $d$. In Figure $8 \mathrm{a}$ it is possible to notice that, except for points 1 and 2, both gradient ( $s=-1$ and $s=1$ ) and PSO algorithms found optimum values for $\varepsilon^{\prime}$ within a range $-27.5<\varepsilon^{\prime}<25.0$. For point number 9, the PSO approach found a solution slightly outside this range. This may be due to a local defect, as pointed out earlier. Regarding the results obtained for points 1 and 2, both of them were edges of the gold coated region of the Otto chip. In this case, the laser footprint was illuminating two different regions, one of which without the gold surface, and thus the measured reflectance was not that described by the model function (1).

The solutions for $\varepsilon^{\prime \prime}$ and $d$ are shown in Figures $8 \mathrm{~b}$ and $8 \mathrm{c}$, respectively. Both solutions from gradient based are well defined for $\varepsilon^{\prime \prime}$, but the PSO solutions switch between those two. A similar switching behavior is observed for the PSO solution for the parameter $d$, as shown in Fig. 8c. One feature also observed in the measured parameters plotted in Fig.8, independent of the method employed, is the existence of oscillations relative to the illumination point on the surface. Those are very likely to occur due to a wobbling effect on the incidence angle, produced by the prism supporting stage, when it is translated across the laser footprint on the surface.

In order to fully analyze the regression strategies, one of the SPR curves was examined in closer detail. Figure 9a shows the SPR data along with the best fit solutions obtained by either gradient or PSO approaches, for point number 10 on the Otto chip. One cannot observe significant differences in this figure. A closer look in the angular region near the resonance valley reveals that PSO has a better fit of the valley relative to the gradient solutions, as can be observed in Fig.9b. Off the resonance angular region, PSO resembles the gradient solution $s=1$, as can be noticed in the plots of Fig. 9c. 


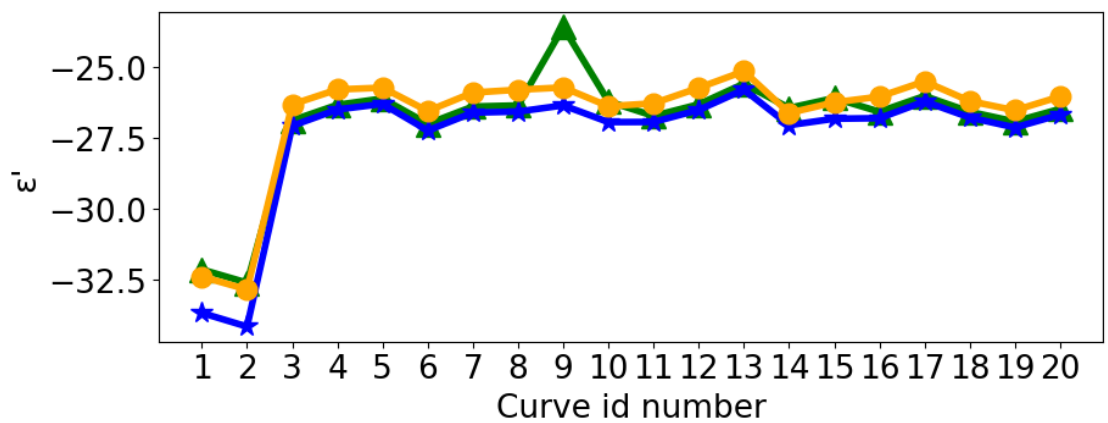

(a) Real permittivity

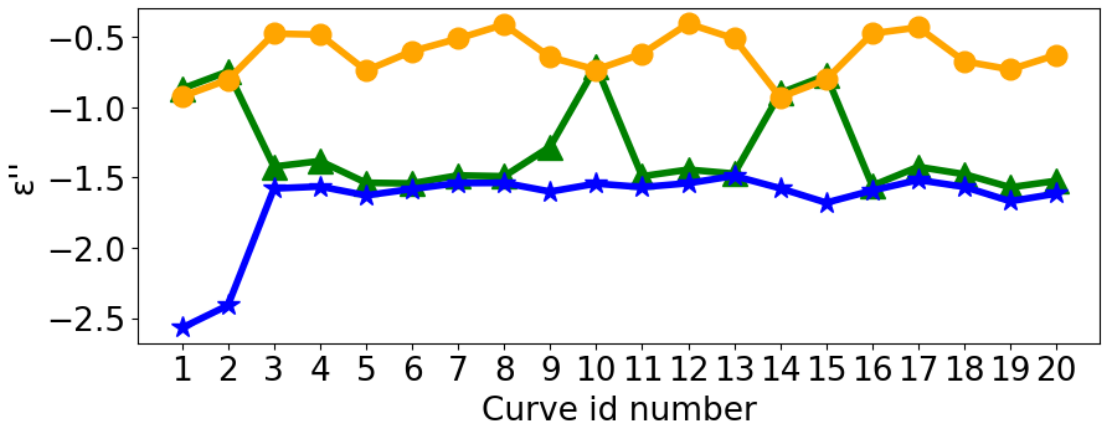

(b) Imaginary permittivity

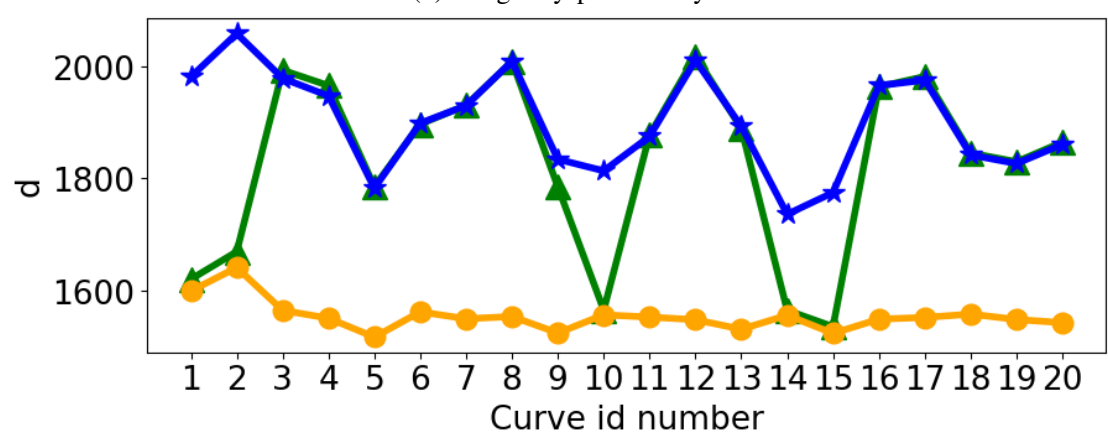

(c) Thickness

$\longrightarrow$ Gradient based $(s=-1) \longrightarrow$ Gradient based $(s=1) \multimap$ PSO

Fig. 8. (a) Real and (b) imaginary parts of the gold complex permittivity and (c) gap thickness obtained from gradient and PSO based approaches.

Brazilian Microwave and Optoelectronics Society-SBMO Brazilian Society of Electromagnetism-SBMag received 20 Oct 2020; for review 5 Nov 2020; accepted 28 Jan 2021 (C) 2021 SBMO/SBMag (cc) BY 


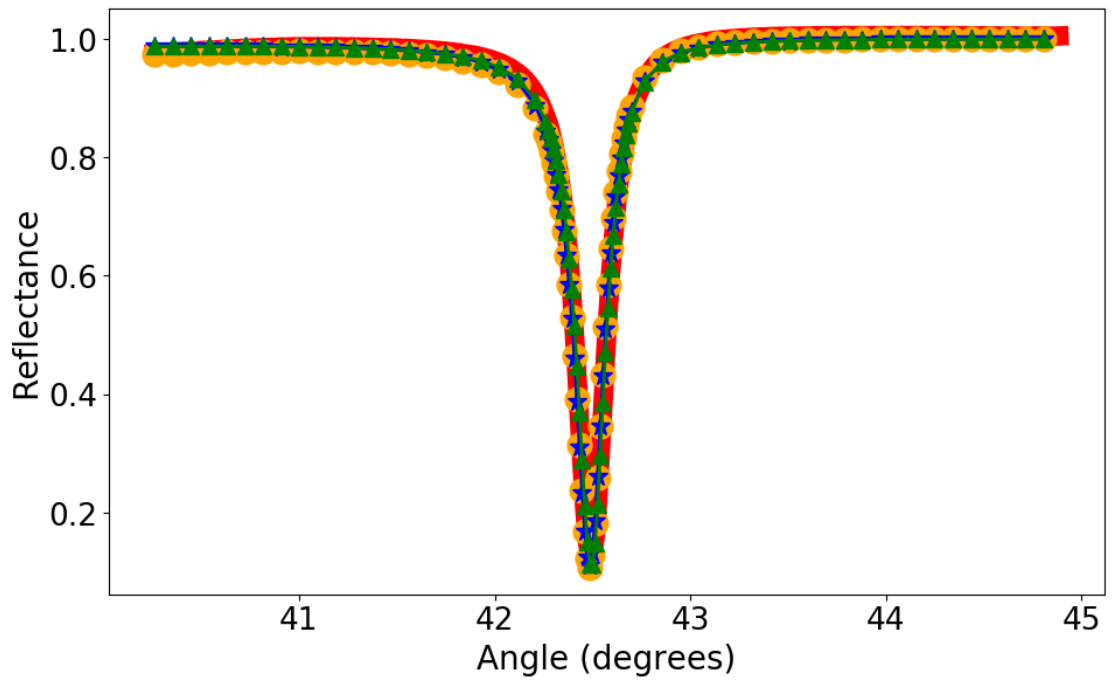

(a)

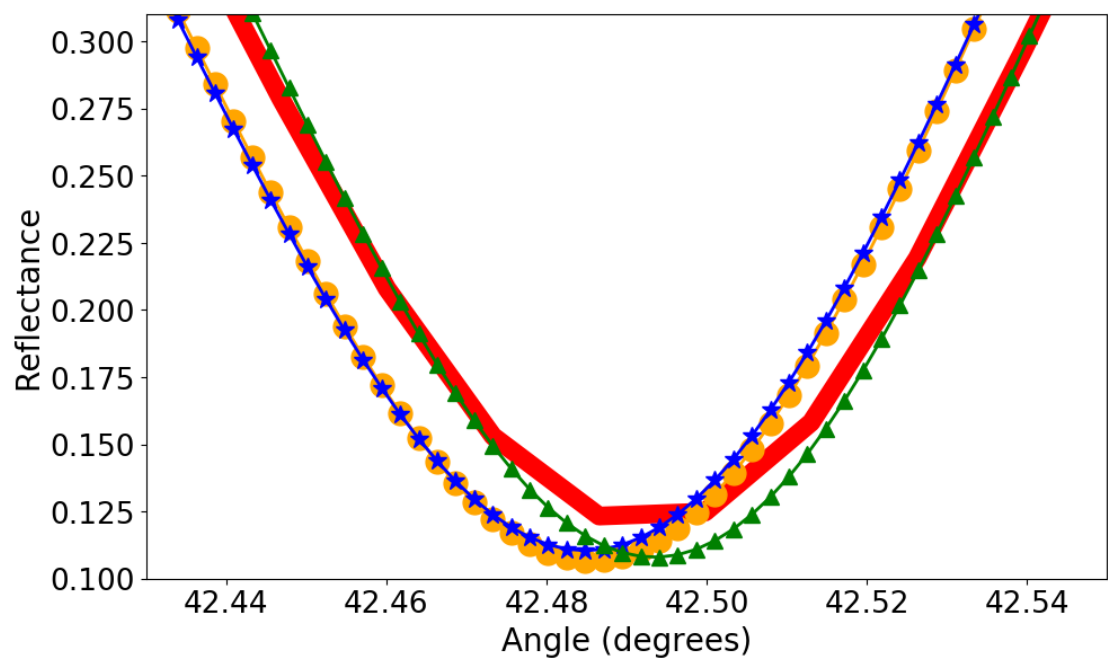

(b)

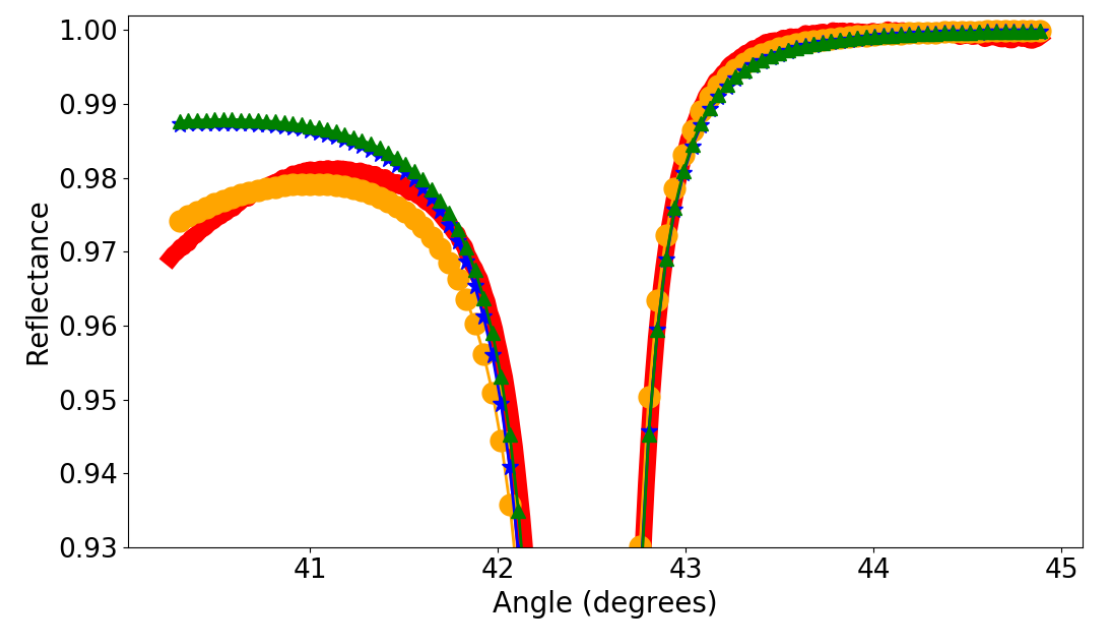

(c)

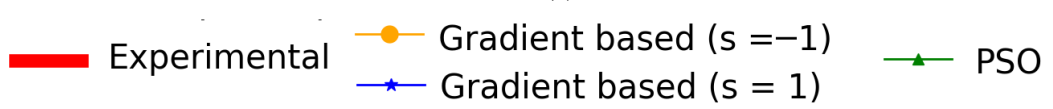

Fig. 9. Experimental and best-fit curves for gradient and PSO based approaches for point number 10 of the Otto chip: (a) Full reflectance curves.(b) Detailed plot of the reflectance curves near the resonance condition. (c)Detailed plot of the reflectance curves off the resonance condition.

Brazilian Microwave and Optoelectronics Society-SBMO Brazilian Society of Electromagnetism-SBMag received 20 Oct 2020; for review 5 Nov 2020; accepted 28 Jan 2021 (C) 2021 SBMO/SBMag (cc) BY 


\section{CONCLUSIONS}

In this work we proposed the use of the Particle Swarm Optimization algorithm for the characterization of Otto chip devices. The strategy was to adapt the principle, which is generally used in optimization problems, to perform regression analysis of data. For Otto chips, SPR reflectance data were analyzed and parameters were determined by minimization of the absolute error of each data set, representing a given point on the Otto chip active area, relative to a three-layer model function. The results, confirmed with $95 \%$ of confidence by the Wilcoxon hypothesis test, indicated that the Clerc PSO strategy can be an effective tool for the regression of SPR curves, as it yields smaller errors when compared to the traditional gradient-based regression approach. This was observed for all 20 curves analyzed in this paper. Another advantage of PSO is the fact that it does not require an initial guess, for running the algorithm. As a result, PSO can be used for batch analysis of Otto chip devices. Because PSO does not require an initial guess, one can use several model functions or combination of these functions, for the analysis of the entire cavity of Otto chip devices. In this way, the $\mathrm{Si}, \mathrm{Au}$, and edge regions on the active area of those devices could be analyzed, with automation of the procedure facilitated by the PSO approach.

The PSO algorithm only returns one solution, by searching on the entire domain for the minimum average absolute error. It was observed, that in this method the solution switches between the two solutions obtained by the gradient method. This behavior should be avoided. One possible way of overcoming this problem is to increase the angular range of the data to be analyzed, so that a unique solution can be found, by looking at other attributes of the reflectance data. In addition, one can also try to minimize the relative, rather than the absolute error. However, this may be a problem, when the reflectance data include very small, almost null, reflectance values, around the minimum reflectance condition. One strategy that could be implemented would be to add an offset to the data, and thus to carry out the regression analysis on the minimum relative error of the data with the added offset. These strategies are currently under investigation.

During the development of this work, PSO and gradient based solutions were executed on different machines and thus a comparison of execution times could not be done. Execution time of the PSO approach was recorded, however, in an Intel Xeon CPU ES-2609 v3, with $1.9 \mathrm{GHz}$ clock, $16 \mathrm{~GB}$ RAM and 64-bit operational system. Each set of 100 PSO executions lasts 79 seconds, considering 30 iterations and 500 particles. This means that each point on the Otto chip surface takes approximately one minute. Simulations were done in interpreted Python and this time will be significantly reduced with a compiled version of the algorithm.

\section{ACKNOWLEDGMENTS}

The authors would like to thank the support provided by the following institutions: FACEPE, Brazil (project number APQ-0145-3.04/17); CNPq, Brazil (scholarships and project number 311625/2019-3), CAPES, Brazil (scholarships and project PRINT/CAPES 88881.311937/2018-01); NATO (SPS project G5640), The Spanish Ministry of Defence (project SensorQ); The Spanish Ministry of Science and Innovation (project RTI2018-099841-B-I00) and National Research Foundation of Korea (project NRF2017R1A2B4005687). 


\section{REFERENCES}

[1] A. Otto, "Excitation of nonradiative surface plasma waves in silver by the method of frustrated total reflection," Zeitschrift für Physik, vol. 216, no. 4, pp. 398-410, 1968.

[2] B. Liedberg, C. Nylander, and I. Lunström, "Surface plasmon resonance for gas detection and biosensing," Sensors and Actuators, vol. 4, pp. 299-304, 1983.

[3] M. T. Flanagan and R. H. Pantell, "Surface plasmon resonance and immunosensors," Electronics Letters, vol. 20, no. 23, pp. 968-970, 1984.

[4] E. Fontana, R. H. Pantell, and S. Strober, "Surface plasmon immunoassay," Applied Optics, vol. 29, no. 31, pp. 46944704, 1990.

[5] S. Sjölander and C. Urbaniczky, "Integrated Fluid Handling System for Biomolecular Interaction Analysis," Analytical Chemistry, vol. 63, no. 20, pp. 2338-2345, 1991.

[6] B. Liedberg, C. Nylander, and I. Lundström, "Biosensing with surface plasmon resonance - how it all started," Biosensors and Bioelectronics, vol. 10, no. 8, pp. i-ix, 1995.

[7] J. Homola, H. Vaisocherová, J. Dostálek, and M. Piliarik, "Multi-analyte surface plasmon resonance biosensing," Methods, vol. 37, no. 1, pp. 26-36, 2005.

[8] P. Tobiška, O. Hugon, A. Trouillet, and H. Gagnaire, "An integrated optic hydrogen sensor based on SPR on palladium," Sensors and Actuators B: Chemical, vol. 74, no. 1-3, pp. 168-172, 2001.

[9] S. K. Mishra and B. D. Gupta, "Surface Plasmon Resonance-Based Fiber-Optic Hydrogen Gas Sensor Utilizing Indium-Tin Oxide (ITO) Thin Films," Plasmonics, vol. 7, no. 4, pp. 627-632, 2012.

[10] E. Kretschmann, "Die Bestimmung optischer Konstanten von Metallen durch Anregung von Oberflächenplasmaschwingungen," Zeitschrift für Physik A Hadrons and nuclei, vol. 241, no. 4, pp. 313-324, 1971.

[11] E. Fontana, J. Kim, I. Llamas-Garro, and G. O. Cavalcanti, "Microfabricated Otto chip device for surface plasmon resonance-based optical sensing," Applied optics, vol. 54, no. 31, pp. 9200-9204, 2015.

[12] E. Fontana, "Matrix formulation for linear and first-order nonlinear regression analysis with multidimensional functions," pp. 01-10, 01 2014. [Online]. Available: http://lsi.fotonica.ufpe.br/fontana/papers/WebPapers/Paper01Fontana2014.pdf

[13] W. P. Chen and J. M. Chen, "Use of surface plasma waves for determination of the thickness and optical constants of thin metallic films," JOSA, vol. 71, no. 2, pp. 189-191, 1981.

[14] J. Salvi and D. Barchiesi, "Measurement of thicknesses and optical properties of thin films from Surface Plasmon Resonance (SPR)," Applied Physics A, vol. 115, pp. 245-255, 2014.

[15] N. Amoosoltani, A. Zarifkar, and A. Farmani, "Particle swarm optimization and finite-difference time-domain (pso/fdtd) algorithms for a surface plasmon resonance-based gas sensor," Journal of Computational Electronics, vol. 18, no. 4, pp. 1354-1364, 2019.

[16] C. Yue, Z. Qin, Y. Lang, and Q. Liu, "Determination of thin metal film's thickness and optical constants based on spr phase detection by simulated annealing particle swarm optimization," Optics Communications, vol. 430, pp. 238-245, 2019.

[17] Y. Sun, C. Haoyuan, X. Wang, and S. Zhan, "Optimization methodology for structural multiparameter surface plasmon resonance sensors in different modulation modes based on particle swarm optimization," Optics Communications, vol. 431, pp. 142-150, 2019.

[18] J. Kennedy and R. Eberhart, "Particle swarm optimization (PSO)," in Proc. IEEE International Conference on Neural Networks, Perth, Australia, pp. 1942-1948, 1995.

[19] A. L. P. da Silva, M. A. Almeida Neto, S. C. Oliveira, G. O. Cavalcanti, and E. Fontana, "A swarm intelligence approach for regression analysis of surface plasmon resonance curves in Otto chips," in 2019 IEEE MTT-S International Microwave and Optoelectronics Conference, pp. 1-3, 2019.

[20] S. Cheng, C. Zhao, J. Wu, and Y. Shi, "Particle swarm optimization in regression analysis: A case study," vol. 7928, pp. 55-63, 2013.

[21] R. C. Jorgenson and S. S. Yee, "A fiber-optic chemical sensor based on surface plasmon resonance," Sensors and Actuators B: Chemical, vol. 12, no. 3, pp. 213-220, 1993.

[22] E. Fontana, "A novel gold-coated multimode fiber sensor," IEEE Transactions on Microwave Theory and Techniques, vol. 50, no. 1, pp. 82-87, 2002.

[23] T. Iqbal and S. Afsheen, "One Dimensional Plasmonic Grating: High Sensitive Biosensor,” Plasmonics, vol. 12, no. 1, pp. 19-25, 2017.

[24] G. O. Cavalcanti, M. A. de Luna, and E. Fontana, "Automated reflectometer for surface plasmon resonance studies in the infrared and its application for the characterization of Pd films," in 2007 SBMO/IEEE MTT-S International Microwave and Optoelectronics Conference, pp. 698-701, 2007. 
[25] E. Fontana and G. O. Cavalcanti, "Maintaining a stationary laser footprint during angular scan in internal-reflection experiments," Appl. Opt., vol. 52, no. 32, pp. 7669-7674, Nov 2013.

[26] M. R. dos Santos, "Analysis of the construction of otto chips by surface plasmon resonance - in portuguese," Master Thesis, Escola Politécnica, Universidade de Pernambuco, Recife, Brazil, 2019.

[27] M. R. N. dos Santos, G. F. Fernandes, G. O. Cavalcanti, E. Fontana, I. Llamas-Garro, Y. Lee, S. Sim, and J. Kim, "Profilometry of the Otto chip by optical reflectometry," YouTube, Apr 2018. [Online]. Available: https://www.youtube.com/watch?v=oyoYfcF2Qv0

[28] E. Fontana, R. H. Pantell, and M. Moslehi, "Characterization of dielectric-coated, metal mirrors using surface plasmon spectroscopy," Appl. Opt., vol. 27, no. 16, pp. 3334-3340, 1988.

[29] Y. Shi and R. Eberhart, "A modified particle swarm optimizer," in 1998 IEEE International Conference on Evolutionary Computation Proceedings. IEEE World Congress on Computational Intelligence (Cat. No.98TH8360), pp. 69-73, May 1998.

[30] M. Clerc and J. Kennedy, "The particle swarm - explosion, stability, and convergence in a multidimensional complex space," IEEE Transactions on Evolutionary Computation, vol. 6, no. 1, pp. 58-73, Feb 2002.

[31] W. Al-Hassan, M. B. Fayek, and S. I. Shaheen, "Psosa: An optimized particle swarm technique for solving the urban planning problem," in 2006 International Conference on Computer Engineering and Systems, pp. 401-405, Nov 2006.

[32] Y. Feng, G. Teng, A. Wang, and Y. Yao, "Chaotic inertia weight in particle swarm optimization," in Second International Conference on Innovative Computing, Informatio and Control (ICICIC 2007), pp. 475-475, Sep. 2007.

[33] J. Xin, G. Chen, and Y. Hai, "A particle swarm optimizer with multi-stage linearly-decreasing inertia weight," in 2009 International Joint Conference on Computational Sciences and Optimization, vol. 1, pp. 505-508, April 2009.

[34] F. Wilcoxon, "Individual comparisons by ranking methods," Biometrics Bulletin, vol. 1, no. 6, pp. 80-83, 1945.

[35] H. W. Lilliefors, "On the Kolmogorov-Smirnov test for normality with mean and variance unknown," Journal of the American Statistical Association, vol. 62, no. 318, pp. 399-402, 1967. 